\title{
Geological Characteristics of the Young Mare Basalts in Chang'e-5 Landing Region, Northern Oceanus Procellarum
}

\author{
YUQI QIAN ${ }^{1,2}$, LONG XIAO $^{1 *}$, JAMES W. HEAD ${ }^{2 *}$
}

${ }^{1}$ School of Earth Sciences, China University of Geosciences, Wuhan, 430074, China

${ }^{2}$ Department of Earth, Environmental, and Planetary Sciences, Brown University, Providence, 02912, USA

Chang'e-5 (CE-5), China's first lunar sample return mission, will collect $\sim 2 \mathrm{~kg}$ of samples from the Northern Oceanus Procellarum landing region, chosen due to the presence of some of the youngest lunar mare basalts [1]. Laboratory analysis and dating of these young mare basalts will significantly improve our understandings of lunar impact flux and chronology, late-stage basalt petrogenesis, the role of Procellarum-KREEP terrain in basalt generation, and lunar thermal evolution. To support laboratory analysis of the returned samples, we undertook a more detailed comprehensive study of the young mare basaltic units in the region.

The young basaltic unit (Em4) forms a smooth mare plain with more abundant $\mathrm{TiO}_{2}$ (6 wt. \%, mean), $\mathrm{FeO}$ (17 wt.\%, mean), and OPX, OLV compared to its Imbrian-aged counterparts. The Em4 unit is the youngest unit in the region, covering all pre-existing features, including highlands, domes, and crater rims. Crater excavation depths suggest that the average thickness of the young basalt unit is $\sim 40 \mathrm{~m}$, and its total volume $\sim 1521 \mathrm{~km}^{3}$. Impact crater size-frequency distribution data suggest an $\sim 1.5 \mathrm{Ga}$ age of emplacement. Rima Sharp, the longest sinuous rille on the Moon, extends across the area. Detailed examination suggests that Rima Sharp is actually composed of two independent sinuous rilles, both of which terminate in the unit.

[1] Qian, Y. et al. (2018) Geology and scientific significane of the Rümker region in northern Oceanus Procellarum: China's Chang'E-5 landing region, J. Geophys. Res., 123, 1407-1430, doi: 10.1029/2018JE005595. 